\title{
LAMOST 多目标光纤光谱仪的研制及试运行
}

\author{
朱永田*，胡中文，王否，王家宁，侯永辉，汤振，戴松新，吴桢，陈忆 \\ 中国科学院南京天文光学技术研究所, 南京 210042 \\ *E-mail: ytzhu@ niaot.ac.cn
}

收稿日期: 2011-09-08; 接受日期: 2011- 09-18; 网络出版日期: 2011-10-19

\begin{abstract}
摘要 LAMOST 望远镜(郭守敬望远镜)配置了 16 台低分辨率多目标光纤光谱仪, 一次曝光可同时获得 4000 个天体的光谱信息. LAMOST 低分辨率光谱仪采用新型体位相全息光栅, 双通道大视场施密特光学系统, 准直光束口径 $200 \mathrm{~mm}$. 每台光谱仪分红、蓝区两个通道, 红蓝区各配置按工作波段优化的 $4 \mathrm{~K} \times 4 \mathrm{~K}$ 科学级 $\mathrm{CCD}$ 芯片, $\mathrm{CCD}$ 采用液氮制冷. 光谱仪波长覆盖范围 370 900 $\mathrm{nm}$, 光谱分辨率 $R=1000 \sim 10000$. 研制完成后的 16 台光谱仪安装于国家天文台兴隆观测站 LAMOST 焦面楼光谱房内, 这些光谱仪已经用于科学试观测并取 得了一批科研成果.
\end{abstract}

关键词光谱仪, 分辨率, 通光效率

PACS: $\quad$ 95.55.Qf, 95.75.Fg, 95.55.Sh

LAMOST 望远镜(大天区面积多目标光纤光谱天 文望远镜 ${ }^{[1]}$ 于 2008 年 10 月 17 日在国家天文台兴隆 观测基地落成, 并于 2010 年 4 月 17 日被冠名为郭守 敬望远镜.

LAMOST 望远镜配备了16台低分辨率光谱仪. LAMOST 低分辨率光谱仪是一种多目标光纤光谱仪. 为获取大样本暗弱天体的光谱信息, LAMOST 低分 辨率光谱仪需实现 4000 个目标同时观测, 波长覆盖 范围 370 900 $\mathrm{nm}$, 光谱分辨率 $R=1000 \sim 10000$, 光谱 仪的光效率高于 $30 \%$.

\section{LAMOST 光谱仪的方案}

\section{1 方案选择}

LAMOST 项目前期, 针对科学目标的要求对低
分辨率光谱仪进行了多种方案的比较研究, 低分辨 率光谱仪第一台样机的实施方案选择的是基于反射 刻划光柱的方案 ${ }^{[2,3]}$. 在样机研制过程中, 又进行了 一种新的体位相全息光栅方案的研究. 鉴于大尺寸 体位相全息光栅的诸多优点, 特别是具有极高的衍 射效率和低杂散光等特性, 备受天文应用的青睐 ${ }^{[4,5]}$, 基于体位相全息光柾的设计被最终确定为 LAMOST 低分辨率光谱仪的实施方案.

设计方案中, 为减少光谱仪的数量, 每台低分辨 率光谱仪应放置尽可能多的光纤. 单台光谱仪最多 能携带的光纤数目 $N$ 主要由光纤芯径 $\varphi$ 、望远镜口径 $D$ 、照相机焦比 $F_{2}$ 、光纤焦比蜕化 $\xi$ 和选择的接收器 尺寸 $s$ 确定. 照相机焦比 $F_{2}$ 直接决定了 LAMOST 每 台光谱仪的光纤数. 在光纤排列方向有如下关系式 ${ }^{[2]}$ :

引用格式: 朱永田, 胡中文, 王否, 等. LAMOST 多目标光纤光谱仪的研制及试运. 中国科学: 物理学 力学 天文学, 2011, 41: 1337-1341 Zhu Y T, Hu Z W, Wang L, et al. Construction and commissioning of LAMOST low resolution spectrographs (in Chinese). Sci Sin Phys Mech Astron, 2011, 41: 1337-1341, doi: 10.1360/132011-927 


$$
s=N\left(\frac{\varphi D F_{2}}{\xi}+d\right),
$$

式中 $d$ 为相邻两行光谱的间隙. LAMOST 光纤较粗, $\mathrm{CCD}$ 像元尺寸较小 $(12 \mu \mathrm{m})$, 如果光谱 6 个像元采样, 则照相机很难设计, 8 10 个像元比较适合.

在色散方向为 ${ }^{[2]}$

$$
s=\frac{R \varphi D}{\xi} F_{2}^{\prime} \times \frac{\Delta \lambda}{\lambda_{b}} .
$$

当接收器尺寸 $s$ 和一次可同时覆盖的光谱范围 $\Delta \lambda$ 一定时, 分辨率 $R$ 越高要求照相机焦比就越强, 或 者说当接收器和照相机焦比一定时, 分辨率越高一 次可同时覆盖的光谱范围就越窄.

LAMOST 低分辨率光谱仪分红、蓝两区, 每区 设有中、低分辨率两种工作模式. 16 台低分辨率光谱 仪可经过网络与望远镜观测控制系统进行接口通讯, 实现远程控制.

\section{2 与国际同类仪器的比较}

表 1 给出了 LAMOST 光谱仪与国际上同类仪器 的比较信息.

LAMOST 与 SDSS 光纤芯对天空的张角相似, 但望远镜口径不同, LAMOST 口径是 SDSS 的 1.6 倍, 从而前者的光纤芯径约是后者的两倍.

LAMOST 与 AAT- $2 \mathrm{dF}$ 望远镜口径相当, 两者光 纤芯对天空张角不同, LAMOST 是 AAT- $2 \mathrm{dF}$ 的 1.78 倍,前者的芯径是后者的两倍多.

LAMOST 同时可观测目标数达到 4000, 也远超

表 1 LAMOST 低分辨率光谱仪和国际同类光谱仪

Table 1 Comparison of LAMOST-LRS and other spectrographs

\begin{tabular}{cccc}
\hline & SDSS & AAT-2dF & LAMOST \\
\hline 通光口径 $(\mathrm{m})$ & 2.5 & 3.9 & 4.0 \\
视场 $\left({ }^{\circ}\right)$ & 2 & 3 & 5 \\
焦比 & 5 & 3.5 & 5 \\
同时观测目标 & 640 & 400 & 4000 \\
焦面比例 & 0.06 & 0.066 & 0.097 \\
$\left(\mathrm{~mm} \mathrm{~s}^{-1}\right)$ & & & \\
光纤芯径 & $0.18 / 3.0$ & $0.14 / 2.16$ & $0.32 / 3.3$ \\
$(\mathrm{~mm} / \mathrm{arcsec})$ & & & \\
焦比蜕化 & $4 / 5$ & $3.15 / 3.5$ & $4 / 5$ \\
光谱范围 $(\mathrm{nm})$ & $390 \sim 910$ & $350 \sim 1000$ & $370 \sim 900$ \\
CCD 规格 & $2048 \times 2048$ & $1024 \times 1024$ & $4096 \times 4096$ \\
象元尺寸 $(\mu \mathrm{m})$ & 24 & 24 & 12 \\
光谱分辨率 & $\sim 2000$ & $500 \sim 2500$ & $1000 \sim 10000$ \\
\hline
\end{tabular}

\section{过 SDSS 和 AAT-2dF.}

以上的三方面因素决定了 LAMOST 低分辨率光 谱仪无论是单体规模或者是整体规模比同类天文仪 器都要大得多.

\section{3 基本设计参数}

图 1 是光学系统的实施方案图, 光学系统采用双 通道全施密特设计, 准直光路和照相机光学都采用 施密特光学系统. 为优化光学系统成像质量, 光学系 统的设计并非经典的施密特系统. 光学系统光束口 径 $200 \mathrm{~mm}$, 由分色镜在 $580 \mathrm{~nm}$ 波长附近将光束分成 红、蓝两个通道, 实现一次曝光全波长覆盖. 光谱仪 的红、蓝两个通道各具有两种工作模式: 低分辨率模 式和中分辨率模式.

低分辨率模式下 $(R=1000 \sim 2000)$, 光栅刻划面尺 寸取为 $\Phi 206 \mathrm{~mm}$, 蓝区光栅工作波段 $\lambda=370 \sim 590 \mathrm{~nm}$, 中心波长取在 $480 \mathrm{~nm}$ 处, 刻线密度 800 线 $/ \mathrm{mm}$; 红区 光栅工作波段 $\lambda=570 \sim 900 \mathrm{~nm}$, 中心波长取在 $735 \mathrm{~nm}$ 处, 刻线密度 540 线 $/ \mathrm{mm}$. 中分辨率模式下 $(R=5000 \sim$ 10000), 光栅刻划面尺寸取为 $\Phi 296 \mathrm{~mm}$, 蓝区光栅工 作波段 $\lambda=510 \sim 540 \mathrm{~nm}$, 中心波长取在 $525 \mathrm{~nm}$ 处, 刻 线密度 2750 线 $/ \mathrm{mm}$; 红区光栅工作波段 $\lambda=830 \sim 890$ $\mathrm{nm}$, 中心波长取在 $860 \mathrm{~nm}$ 处, 刻线密度 1680 线 $/ \mathrm{mm}$. 照相机焦距 $270 \mathrm{~mm}$, 焦比 $f / 1.3$, 焦面前加场镜得平 像面, 具体见表 2 和 3.

\section{2 光学系统通光效率}

低分辨率光谱仪中体位相全息光栅的应用提高 了光学系统的通光效率, 2008 年对光学系统的效率进 行了系统的测试, 图 2 是光谱仪在不同波长(单位: $\mathrm{nm}$ )处的通光效率百分比的实测结果, 测试的数据不 包含光纤透过率和 CCD 量子效率.

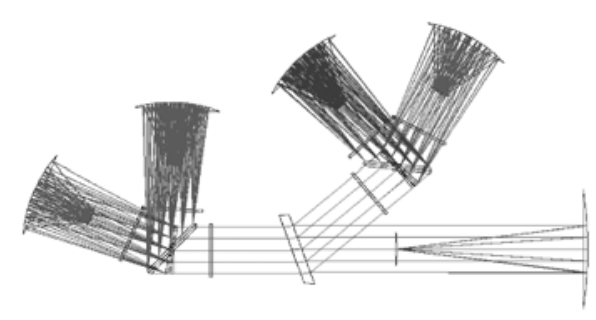

图 1 光谱仪光学系统方案

Figure 1 Optical layout of LAMOST LRS. 
表 2 低分辨率光谱仪蓝区性能参数

Table 2 Parameters for blue channel of LRS

\begin{tabular}{|c|c|c|c|}
\hline \multicolumn{2}{|c|}{ 光㮽参数 } & \multicolumn{2}{|l|}{ 光学系统参数 } \\
\hline 线密度 $\left(\mathrm{g} \mathrm{mm}^{-1}\right)$ & 800 & 准直光束口径 $(\mathrm{mm})$ : & 200 \\
\hline 闪耀角 $\left(^{\circ}\right)$ & 11.07 & 焦比 & $f / 4$ \\
\hline 级次 & 1 & $\begin{array}{c}\text { 望远镜口径 }(\mathrm{mm}): \\
\text { 焦比 }\end{array}$ & $\begin{array}{l}4000 \\
f / 5\end{array}$ \\
\hline 入射角 $\left(^{\circ}\right)$ & 8.47 & 光纤芯径 $(\operatorname{arcsec} / \mathrm{mm})$ & $3.3 / 0.32$ \\
\hline 衍射角 $\left(^{\circ}\right)$ & 13.67 & $\begin{array}{l}\text { 光纤焦比退化 } \\
\text { 相机焦距 }(\mathrm{mm}):\end{array}$ & $\begin{array}{c}0.8 \\
\mathrm{f} 270\end{array}$ \\
\hline$\lambda_{\min }(\mathrm{mm})$ & 370 & 分辨率 $X$ 视宁度 $(R \times \phi)$ & 3200 \\
\hline$\lambda_{\mathrm{b}}(\mathrm{mm})$ & 479.5 & $\begin{array}{c}\text { 狭缝投影比: } W(\text { 狭缝 }) / \\
W(\text { 像 })\end{array}$ & 2.911 \\
\hline $\begin{array}{c}\mathrm{CCD} \\
\text { 像素大小 }(\mu \mathrm{m})\end{array}$ & $\begin{array}{c}4096 \times 4096 \\
12\end{array}$ & \begin{tabular}{||c} 
像面比例尺 \\
$(\operatorname{arcsec} / \mathrm{mm})$ 在 $\mathrm{CCD}$ 上.
\end{tabular} & 30.020 \\
\hline
\end{tabular}

表 3 低分辨率光谱仪红区性能参数

Table 3 Parameters for red channel of LRS

\begin{tabular}{|c|c|c|c|}
\hline \multicolumn{2}{|c|}{ 光栅参数 } & \multicolumn{2}{|l|}{ 光学系统参数 } \\
\hline 线密度 $\left(\mathrm{g} \mathrm{mm}^{-1}\right)$ & 540 & 准直光束口径 $(\mathrm{mm})$ : & 200 \\
\hline 闪耀角 $\left(^{\circ}\right)$ & 11.53 & 焦比 & $f / 4$ \\
\hline \multirow[t]{2}{*}{ 级次 } & 1 & 望远镜口径(mm): & 4000 \\
\hline & & 焦比 & $f / 5$ \\
\hline 入射角 $\left(^{\circ}\right)$ & 8.93 & 光纤芯径( $\operatorname{arcsec} / \mathrm{mm})$ & $3.3 / 0.32$ \\
\hline \multirow[t]{2}{*}{ 衍射角 $\left(^{\circ}\right)$} & 14.13 & 光纤焦比退化 & 0.8 \\
\hline & & 相机焦距(mm) & $\mathrm{f} 270$ \\
\hline$\lambda_{\min }(\mathrm{nm})$ & 570 & 分辨率 $X$ 视宁度 $(R \times \phi)$ & 3334 \\
\hline$\lambda_{\mathrm{b}}(\mathrm{nm})$ & 739.2 & 狭缝投影比: $W$ (狭缝)/ & 2909 \\
\hline$\lambda_{\max }(\mathrm{nm})$ & 900 & $W$ (像) & 2.909 \\
\hline $\mathrm{CCD}$ & $4096 \times 4096$ & 像面比例尺 & 29997 \\
\hline 像素大小 $(\mu \mathrm{m})$ & 12 & $(\operatorname{arcsec} / \mathrm{mm})$ 在 CCD 上. & \\
\hline
\end{tabular}

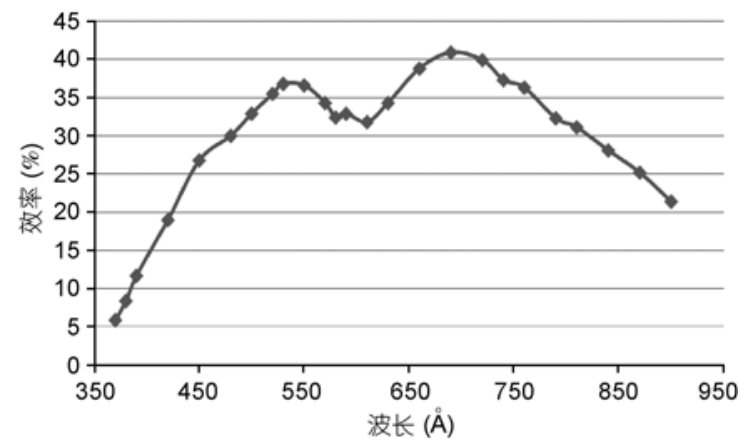

图 2 不同波长处光谱仪通光效率百分比图 Figure 2 Throughput of LAMOST LRS.

\section{3 试运行调整及初步观测结果}

\section{1 运行状态}

2008 年 16 台光谱仪完成全部装调和测试 ${ }^{[6]}$, 图 3 是装调后的光谱仪群. 随后每台光谱仪增加单独的 罩壳, 用于防尘和改善温度稳定性. 2010 年夏季, 光 谱仪进行了系统的优化, 大幅降低了光学系统的杂 散光水平, 优化后用单色光测试, 光谱仪光学系统的 杂散光水平小于 $5 \%$.

\section{2 试观测}

在 2009 年冬天的科学试观测中发现了一批新的 类星体、贫金属恒星的候选体，在仙女星系外围中新 发现的星系状星云等新天体 ${ }^{[7]}$, 在这里对这些观测结 果我们不做具体的描述, 仅给出一部分观测的晨昏 蒙影的天光光谱和天体光谱. 图 4 给出了一根光纤的 晨昏蒙影天光光谱(仅进行波长定标). 图 5 给出了部 分贫金属恒星候选体的天体光谱. 图 6 给出了部分观 测到的行星状星云的光谱.

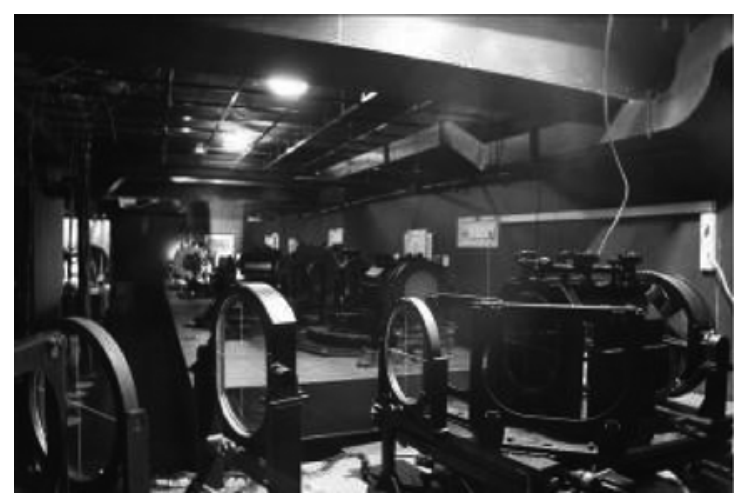

图 3 兴隆观测站 LAMOST 光谱房光谱仪群

Figure 3 LAMOST LRSs on site.

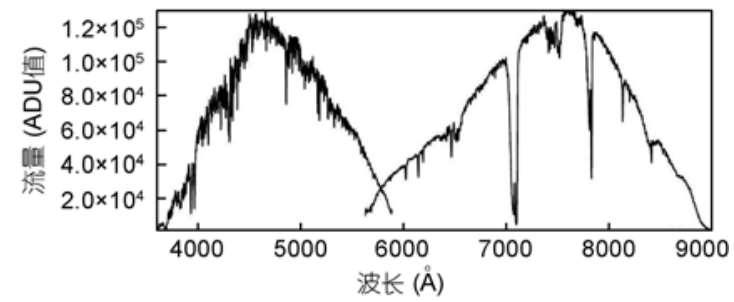

图 4 晨昏蒙影时的一维天光光谱

抽取的一维谱仅进行了波长定标, 但未进行流量定标

Figure 4 The 1-D spectrum of twilight sky. The extracted spectrum is wavelength-calibrated, no flux-calibrated. 


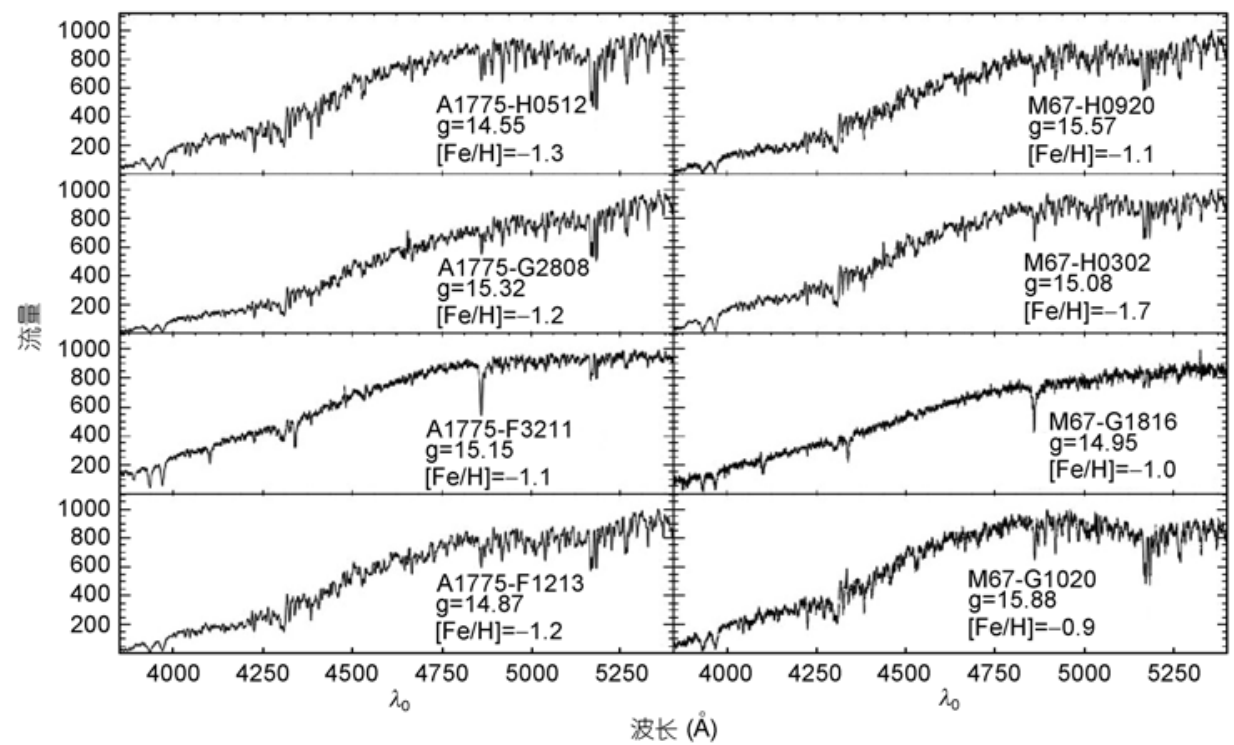

图 5 部分观测到的贫金属恒星候选体的光谱

图中给出了LAMOST ID、SDSS 的 $\mathrm{g}$ 波段星等和 $[\mathrm{Fe} / \mathrm{H}]$

Figure 5 LAMOST spectra of the metal-poor candidates. LAMOST ID, SDSS g-band magnitude are shown, together with the measured [Fe/H].

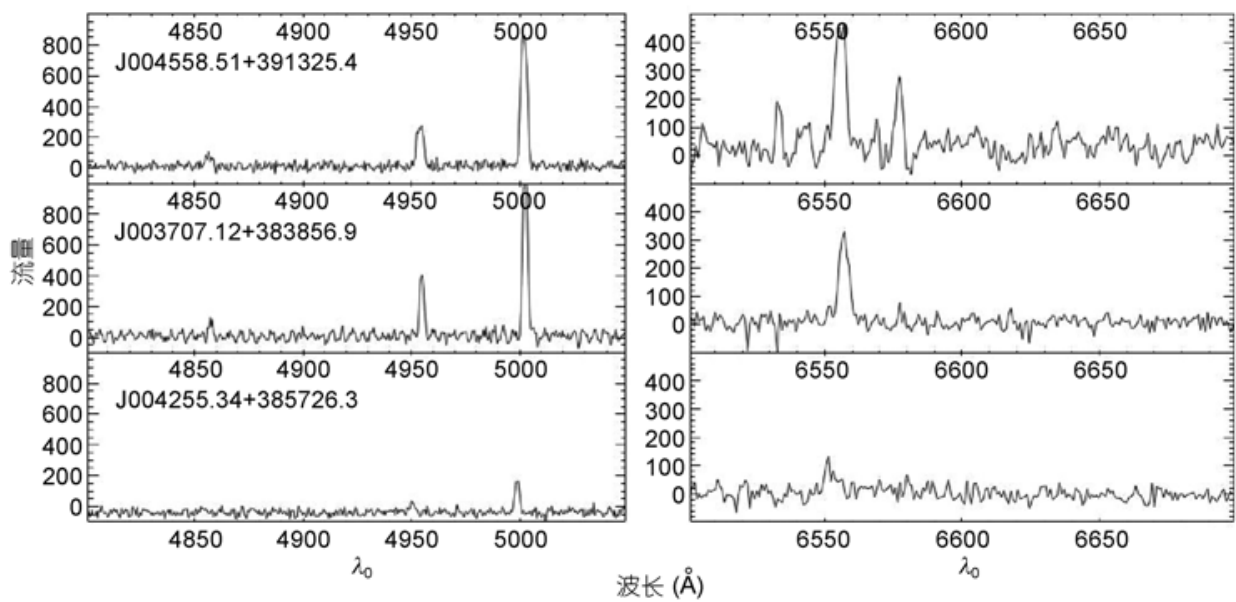

图 6 在分辨率为 2000 模式下，部分新发现行星状星云的观测结果

Figure 6 Some LAMOST spectra of newly discovered PNe in $R=2000$ mode.

\section{参考文献}

1 Wang S G, Su D Q, Chu Y Q, et al. Special configuration of a large Schmidt telescope for extensive astronomical spectroscopic observation. Appl Opt, 1996, 35: 5155-5161

2 Zhu Y T, Xu W L. Low resolution spectrograph for LAMOST. Proc SPIE, 2000, 408: 141-147

3 Zhu Y T, Lemaitre G R. The LAMOST multi-object spectrographs with aspherized gratings. Proc SPIE, 2003, 4841: 1127-1133

4 Smith G A, Saunders W, Bridges T, et al. AAOmega: A multi purpose fiber-fed spectrograph for the AAT. Proc SPIE, 2004, 5492: 410-420

5 Saunders W, Bridges T, Gillingham P, et al. AAOmega: A scientific and optical overview. Proc SPIE, 2004, 5492: 389-400

6 Cui X Q. Preparing first light of LAMOST. Proc SPIE, 2008, 7012: 701204 
7 Yuan H B, Liu X W, Huo Z Y, et al. New planetary nebulae in the outskirts of the Andromeda Galaxy discovered with the Guoshoujing Telescope (LAMOST). Res Astron Astrophys, 2010, 10: 599-611

\title{
Construction and commissioning of LAMOST low resolution spectrographs
}

\author{
ZHU YongTian*, HU ZhongWen, WANG Lei, WANG JiaLing, HOU YongHui, \\ TANG Zhen, DAI SongXin, WU Zhen \& CHEN Yi \\ Nanjing Institute of Astronomical Optics \& Technology, Chinese Academy of Sciences, Nanjng 210042, China
}

There are 16 low resolution multi-objects fiber-fed spectrographs (LRS) for the LAMOST project. The spectrographs are of double-beam full Schmidt design by using volume phase holographic gratings. Each spectrograph will be accommodating 250 fibers of 320 micron in diameter which corresponds $3.3 \mathrm{arcsec}$. The $200 \mathrm{~mm}$ diameter collimated beam is split into two separate channels by a clichroic filter. The blue channel is optimized for $370-590 \mathrm{~nm}$, and the red channel for $570-900 \mathrm{~nm}$. Spectra are focused onto $4 \mathrm{~K} \times 4 \mathrm{~K} \mathrm{CCD}$ using fast camera with focal ratio of 1.25 . The spectrum resolution is 1000 for low resolution mode. Resolution of 5000 is available by automatic switching to different gratings and by changing camera working angles. The resolution could be doubled by restricting the width of a slit to half the size of fiber diameter. These spectrographs are installed under focal plane of LAMOST at Xinglong observatory. Some of the results obtained during commissioning period are reported.

spectrograph, spectal resolution, efficiency

PACS: 95.55.Qf, 95.75.Fg, 95.55.Sh

doi: $10.1360 / 132011-927$ 